\section{Saúde dos adolescentes da coorte de nascimentos de São Luís, Maranhão, Brasil, 1997/1998}

\author{
Health of adolescents in the 1997/1998 birth \\ cohort in São Luís, Maranhão State, Brazil
}

\author{
Salud de los adolescentes de la cohorte de \\ nacimientos de São Luís, Maranhão, \\ Brasil, 1997/1998
}

Vanda Maria Ferreira Simões 1

Rosângela Fernandes Lucena Batista 1

Maria Teresa Seabra Soares de Britto e Alves 1

Cecilia Cláudia Costa Ribeiro 1

Erika Bárbara Abreu Fonseca Thomaz 1

Carolina Abreu de Carvalho 1

Antônio Augusto Moura da Silva 1

\section{Resumo}

O objetivo foi estimar a prevalência de indicadores de saúde de adolescentes em São Luís, Maranhão, Brasil, em 2016. Foram estudadas condições sociodemográficas, hábitos de vida, composição corporal, qualidade do sono, atividade física, habilidade cognitiva e risco de suicídio de 2.515 adolescentes com 18 e 19 anos. Os adolescentes pertencem à coorte de nascimento 1997/1998 ou foram incluídos, retrospectivamente, utilizando-se o banco do SINASC (Sistema de Informações sobre Nascidos Vivos). Os adolescentes eram, principalmente, da classe econômica $C$ (50,1\%), 69,5\% estudavam, 40,3\% trabalhavam e 25,2\% não estudavam nem trabalhavam; 60,3\% já foram vitimas de assalto e 48,7\% tinham pais separados. Apresentaram padrão de consumo nocivo, excessivo ou provável dependência de álcool 19,4\%, 19,1\% usaram ou estavam usando drogas ilicitas, 53,7\% tinham qualidade de sono ruim, 40,8\% referiram dor de cabeça frequente, 34,3\% declararam tempo de tela por dia maior que cinco horas e 4,1\% apresentaram alto risco para suicídio. A prevalência de hipertensão arterial foi de $12 \%$ e de obesidade, 6\%. As meninas eram mais inativas $(80,7 \%)$ e apresentaram maior percentual de gordura corporal alto $(15,8 \%) e$ muito alto (21,5\%), já os meninos tiveram maior prevalência de hipertensão arterial (21,2\%) e menor prevalência de inatividade física (40,9\%). As elevadas prevalências de fatores de risco à saúde aumentam a vulnerabilidade dos adolescentes, expondo estes individuos precocemente a fatores que levam ao acometimento cada vez mais cedo de doenças e agravos à saúde.

Adolescente; Vulnerabilidade Social; Estado Nutricional; Transtornos Mentais; Sono

\author{
Correspondência \\ V. M. F. Simões \\ Departamento de Saúde Pública, Universidade Federal do \\ Maranhão. \\ Rua Barão de Itapary 155, São Luís, MA 65020-070, Brasil. \\ vandamfsimoes@gmail.com \\ 1 Departamento de Saúde Pública, Universidade Federal do \\ Maranhão, São Luís, Brasil.
}




\section{Introdução}

Indicadores de saúde de crianças e adolescentes repercutem em muitos aspectos da vida futura, como a alimentação, autoimagem, saúde individual, valores, preferências e desenvolvimento psicossocial 1 .

A adolescência é um período crítico de transição entre a infância e a vida adulta, caracterizado pela vulnerabilidade física, social e psicológica. Nessa fase, é comum a adoção de hábitos e comportamentos de risco relacionados ao estilo de vida que impactam na morbimortalidade atual e futura desses indivíduos 2,3. Dentre os comportamentos mais prevalentes, pode-se citar o consumo de bebidas alcoólicas, energéticas, uso de drogas ilícitas, tabagismo, sedentarismo, hábitos alimentares inadequados e baixa qualidade do sono. Outros aspectos que também podem interferir de forma significativa no estado de saúde e qualidade de vida na adolescência são a exposição a conflitos familiares, bullying, evasão e reprovação escolar, além de baixo nível socioeconômico 1,2.

Como resultado desses comportamentos e exposições têm sido reportadas prevalências elevadas de diversos agravos e doenças entre adolescentes. Recentemente, o Estudo de Riscos Cardiovasculares em Adolescentes (ERICA), que avaliou adolescentes de 12 a 17 anos, encontrou altas prevalências no Brasil e no Nordeste, respectivamente, de hipertensão $(9,6 \% ; 8,4 \%) 4$, obesidade $(8,4 \% ; 7,4 \%) 4$, HDLcolesterol baixo $(46,8 \% ; 51,6 \%) 5$, hipercolesterolemia $(20,1 \% ; 18,8 \%) 5$, hipertrigliceridemia $(7,8 \%$; $9,4 \%) 5$, transtorno mental comum $(30 \% ; 30,2 \%) 6$, elevado tempo de tela $(51,8 \% ; 69,2 \%$ - duas horas ou mais) 7 , inatividade física no lazer $(54,3 \% ; 55,7 \%) 8$, uso de bebidas alcoólicas $(21 \% ; 16,7 \%)$, dentre outros 3 . Para diversos desses desfechos foram observadas diferenças entre os sexos e por região do Brasil.

A exposição à violência comunitária é outro fator muito presente na vida dos jovens brasileiros. Estudos realizados em São Gonçalo (Rio de Janeiro) 10 e São Leopoldo (Rio Grande do Sul) 11 apontam que $93,2 \%$ e 90,2\% dos adolescentes entrevistados já haviam sofrido pelo menos um episódio de violência comunitária, respectivamente. No estudo realizado em São Leopoldo, 20,8\% dos adolescentes já tinham sido assaltados 11. A exposição à violência comunitária representa um fator de risco à saúde dos adolescentes, aumentando a chance do desenvolvimento de transtornos mentais 12 .

Nesse contexto, a elevada prevalência de fatores de risco cardiovasculares e para outras morbidades já é uma realidade entre os adolescentes brasileiros. Estudos que avaliem os indicadores de saúde nessa população são fundamentais para o estabelecimento de políticas públicas eficazes na promoção da saúde 3 .

No presente trabalho, pretende-se estimar a prevalência de indicadores de saúde, tais como condições sociodemográficas, hábitos de vida, composição corporal, qualidade do sono, atividade física, habilidade cognitiva e transtornos mentais entre adolescentes de uma coorte de nascimento realizada em uma cidade do Nordeste brasileiro, além de identificar diferenças nas prevalências de alguns indicadores entre os sexos. A avaliação da composição corporal por métodos de alta validade, da qualidade do sono, da habilidade cognitiva, do uso de bebidas energéticas e exposição à violência comunitária são aspectos ainda pouco explorados em grandes estudos entre adolescentes. Portanto, os indicadores de saúde analisados neste trabalho têm o potencial de contribuir para a concepção e/ou redirecionamento de políticas públicas voltadas para adolescentes, um público ainda pouco alcançado por ações específicas, apesar dos inúmeros fatores de risco presentes nesta faixa etária.

\section{Métodos}

Estudo transversal aninhado a um estudo de coorte, com indivíduos nascidos na cidade de São Luís, Maranhão, Brasil. Essa coorte está incluída no consórcio de coortes (Coortes RPS - consórcio de coortes brasileiras de nascimento de Ribeirão Preto, Pelotas e São Luís) desenvolvido pela Universidade Federal do Maranhão (UFMA), Faculdade de Medicina de Ribeirão Preto (Universidade de São Paulo, USP) e Universidade Federal de Pelotas (UFPel). Neste artigo, utilizaram-se dados de São Luís, que é a capital do Estado do Maranhão, situada em uma ilha localizada no litoral norte do estado, na Região Nordeste do Brasil.

No momento inicial da coorte, por ocasião do nascimento dos seus membros, o estudo foi conduzido em dez hospitais da cidade, públicos e privados, de março de 1997 a fevereiro de 1998. A amostra, 
de base populacional dos nascimentos de São Luís, correspondeu a 96,3\% dos nascimentos no período estudado, excluindo-se os nascimentos não hospitalares e os ocorridos em hospitais com menos de 100 partos por ano. Utilizou-se amostragem sistemática com estratificação proporcional de acordo com o número de nascimentos em cada maternidade, de um em cada sete partos. Obteve-se um total de 2.831 nascimentos. Excluídos os não residentes em São Luís, gemelares e natimortos, a amostra final foi de 2.443 nascimentos, com $5,8 \%$ de perdas devido a recusas ou alta precoce 13 .

Essa coorte foi acompanhada aos 7-9 anos e novamente aos 18-19 anos. Neste artigo foram utilizados os dados do segundo seguimento, realizado com os indivíduos aos 18-19 anos, em 2016. Todos os participantes incluídos na fase inicial do estudo foram buscados nas quatro Juntas de Alistamento Militar na Ilha de São Luís, no censo escolar de 2014 e em universidades, totalizando 684 participantes. O foco da avaliação foram os desfechos relacionados à nutrição, à composição corporal, aos precursores de doenças crônicas, à saúde mental e ao capital humano.

Visando a aumentar o poder da amostra e prevenir perdas futuras, a coorte foi aberta para incluir outros indivíduos nascidos em São Luís, no ano de 1997. A primeira etapa de busca ocorreu a partir de sorteio utilizando-se o banco do SINASC (Sistema de Informações sobre Nascidos Vivos). Os seguintes critérios foram levados em consideração para o cadastro: ter nascido em maternidade, na cidade de São Luís, no ano de 1997. Com base nessa listagem foi feito um sorteio aleatório, obtendose um total de 4.593 nascidos em 1997 na cidade de São Luís. Desse total, foi possível fazer contato telefônico ou pessoal com 1.133. Em uma segunda etapa, 695 voluntários nascidos no mesmo ano foram identificados nas escolas, universidades e pelas mídias sociais, totalizando 1.828 adolescentes. Os membros do componente retrospectivo da coorte foram submetidos aos mesmos testes e questionários que os demais participantes da coorte original. Além disso, foi aplicado um questionário às mães desses adolescentes para coletar dados perinatais de forma retrospectiva. A amostra total foi de 2.515 participantes, residentes em São Luís.

A coleta de dados ocorreu nas dependências da UFMA. Foram contratados profissionais da área da saúde, que foram treinados para a aplicação dos questionários da pesquisa ou manuseio dos equipamentos. Foram usados os seguintes instrumentos e variáveis:

(1) Características socioeconômicas: sexo (masculino/feminino); classe econômica pelo Critério de Classificação Econômica do Brasil 2016 (A, B1, B2, C1, C2, D/E, sendo a classe A a mais rica e escolarizada e a classe D/E a mais pobre e menos instruída - Associação Brasileira de Empresas de Pesquisa, http://www.abep.org/criterio-brasil); estuda atualmente (sim/não); já trabalhou alguma vez (sim/ não); trabalha atualmente (sim/não); não trabalha, nem estuda (sim/não); tem alguma religião (sim/ não); cor da pele (branca, preta/negra, parda/mulata/cabocla/morena); pais separados ou divorciados (sim/não); chefe de família (pai/mãe/avós/próprio adolescente/outro); plano de saúde (sim/não).

(2) Hábitos de vida e eventos estressores: tempo de tela - televisão, videogame, celular, tablet e computador (<2h; 2-4,9h; 5+); assaltado alguma vez (sim/não); alguma vez sentiu insegurança no bairro (sim/não); fumo atual independentemente da frequência e/ou quantidade (sim/não); hábito de fumar no passado pelo menos uma vez por semana (sim/não); questionário autoaplicado sobre o uso de drogas ilícitas (nunca usou/já usou ou usa atualmente); uso de bebidas energéticas (sim/não); presença de dores de cabeça frequentes (sim/não); padrão de consumo de álcool: baixo/abstinência (0-7), risco (8-15), nocivo (16-19) e provavelmente dependente (20-40), utilizando-se o instrumento Alcohol Use Disorder Identification Test (AUDIT) 14.

(3) Hipertensão arterial: PAS (pressão arterial sistólica) $\geq 130 \mathrm{mmHg}$ e/ou PAD (pressão arterial diastólica) $\geq 80$, calculada com base na média das três medidas aferidas no aparelho Omron HEM 742INT (Omron, São Paulo, Brasil), obtidas após pelo menos cinco minutos em repouso.

(4) Saúde mental: risco de suicídio (baixo/moderado/alto); depressão, que inclui o diagnóstico de episódio depressivo maior atual e recorrente (sim/não); transtorno bipolar (sim/não); transtorno de ansiedade generalizada atual (sim/não), utilizando-se o questionário MINI (Mini International Neuropsychiatric Interview - Brazilian version 5.0.0 - DSM IV - Diagnostic and Statistical Manual of Mental Disorders) 15. Todos os participantes que apresentaram algum problema de saúde mental identificado pelo MINI foram encaminhados para acompanhamento em ambulatório especializado.

(5) Sono: qualidade do sono (boa/ruim) e sonolência excessiva, utilizando-se dois instrumentos validados no Brasil: Índice de Qualidade de Sono de Pitsburg (IQSP) 16 e Epworth Sleep Scale (ESS) 17. 
Pontuação de 0 a 4 é considerada boa qualidade do sono e acima de 5 pontos esta qualidade é ruim. A sonolência diurna excessiva foi considerada para escore $\geq 99$.

(6) Habilidade cognitiva: quociente de inteligência (QI) total, abaixo da média (66 a 90 pontos), inteligência média (91 a 110), acima da média (maior que 110), avaliada por meio da Weschler Adult Intelligence Scale - WAIS III 18. Esse instrumento foi aplicado exclusivamente por profissionais graduados em psicologia.

(7) Composição corporal: percentual de gordura corporal obtido por pletismografia por deslocamento de ar, no equipamento BOD POD Gold Standard da marca COSMED (COSMED Metabolic Company, Roma, Itália) e classificado de acordo com Lohman 19 (muito baixo/baixo/ótimo/moderadamente alto/alto/muito alto). $\mathrm{O}$ peso dos adolescentes foi avaliado na balança de alta precisão acoplada ao BOD POD e a altura com base no estadiômetro AlturaExata (Belo Horizonte, Brasil). $\mathrm{O}$ estado nutricional dos adolescentes foi avaliado pelo indicador índice de massa corporal (IMC)/ Idade, conforme valores estabelecidos nas curvas de crescimento da Organização Mundial da Saúde (OMS) 20. Considerou-se baixo peso para valores menores que -2 desvios padrão(DP), sobrepeso para valores maiores que $>1 \mathrm{DP}$ e obesidade $>2 \mathrm{DP}$.

(8) Atividade física de lazer: atende/não atende à recomendação. Avaliada por intermédio do Inquérito de Atividade Física, elaborado baseando-se em uma adaptação do Self Administered Physical Activity Checklist (SAPAC) 21. Trata-se de um instrumento validado por meio do qual é possível estimar a prática de atividade física de lazer pela frequência e duração das atividades realizadas. Com base nas informações obtidas no Inquérito de Atividade Física, o índice de atividade física semanal foi calculado multiplicando-se o tempo gasto em cada atividade pelo número de dias (frequência) em que ela foi realizada. Sallis et al. 21 definem os equivalentes metabólicos (METs) gastos em cada atividade física que compõem o Inquérito de Atividade Física. Cada atividade física foi classificada como moderada ou intensa, de acordo com o número de MET gastos. De acordo com Ainsworth et al. 22, atividades com gasto de 4,5 a 6,0 MET são moderadas e aquelas com gasto maior ou igual a 6,0 MET são intensas. Com base nisso, considerou-se que atenderam à recomendação de prática de atividade física aqueles que praticaram pelo menos 75 minutos de atividade física intensa ou 150 minutos de atividade moderada por semana, conforme recomendado pela OMS 23.

\section{Processamento e análise estatística de dados}

Os dados coletados foram digitados on-line utilizando-se o sistema RedCap (https://www.project-re dcap.org/). A análise estatística foi feita no programa Stata 12.0 (https://www.stata.com). Na análise descritiva foram calculadas as frequências absolutas e porcentagens. $O$ teste do qui-quadrado foi usado para calcular a diferença entre as proporções de acordo com o sexo. O nível de significância foi fixado em 0,05 e o intervalo de 95\% de confiança (IC95\%).

\section{Aspectos éticos}

O estudo atendeu aos critérios da Resolução no 466/2012 do Conselho Nacional de Saúde e da Norma Operacional no 001/2013. Os adolescentes que concordaram em participar da pesquisa assinaram o Termo de Consentimento Livre e Esclarecido (TCLE). O projeto e o TCLE foram aprovados pelo Comitê de Ética em Pesquisa do Hospital Universitário, parecer consubstanciado no 1.302 .489 de 29 de outubro de 2015. 


\section{Resultados}

Foram avaliados 2.515 adolescentes. Os resultados foram apresentados estratificados por sexo. Estavam estudando 69,5\% dos adolescentes, 40,3\% trabalhavam no momento da coleta, porém, 25,2\% não trabalhavam, nem estudavam. A condição "nem-nem" foi mais prevalente nas meninas. A maior parte dos adolescentes era da classe econômica C (50,1\%), apenas 20,2\% possuíam plano de saúde, $40,3 \%$ tinham o pai como chefe da família e em 32,9\% a mãe exercia este papel. Quase a metade dos adolescentes (48,7\%) tinha pais separados. A exposição à violência comunitária foi elevada, sendo que $60,3 \%$ dos adolescentes já tinham sido assaltados alguma vez na vida, sendo esta ocorrência maior em meninos (63,7\% vs. 57,2\%). Apesar disso, as meninas foram as que mais referiram insegurança no bairro $(81,4 \%$ vs. $65,9 \%)$ (Tabela 1$)$.

\section{Tabela 1}

Características sociodemográficas e insegurança dos adolescentes da Coorte RPS (consórcio de coortes de nascimento brasileiras em Ribeirão Preto, Pelotas e São Luís) por sexo. São Luís, Maranhão, Brasil, 2016.

\begin{tabular}{|c|c|c|c|c|c|c|c|}
\hline \multirow[t]{2}{*}{ Variáveis } & \multicolumn{2}{|r|}{ Total } & \multicolumn{2}{|c|}{ Masculino } & \multicolumn{2}{|c|}{ Feminino } & \multirow[t]{2}{*}{ Valor de $p$} \\
\hline & $\mathbf{n}$ & $\%(I C 95 \%)$ & $\mathbf{n}$ & $\%$ & $\mathbf{n}$ & $\%$ & \\
\hline Estuda atualmente & & & & & & & 0,233 \\
\hline Não & 767 & $30,5(28,7-32,3)$ & 351 & 29,4 & 416 & 31,5 & \\
\hline Sim & 1.748 & $69,5(67,7-71,3)$ & 845 & 70,6 & 903 & 68,5 & \\
\hline Já trabalhou alguma vez na vida & & & & & & & $<0,001$ \\
\hline Não & 1.530 & $60,8(58,9-62,7)$ & 634 & 53,0 & 896 & 67,9 & \\
\hline Sim & 985 & $39,2(37,3-41,1)$ & 562 & 47,0 & 423 & 32,1 & \\
\hline Trabalha atualmente & & & & & & & $<0,001$ \\
\hline Não & 588 & $59,7(56,6-62,8)$ & 338 & 60,1 & 250 & 59,1 & \\
\hline Sim & 397 & $40,3(37,2-43,4)$ & 224 & 39,9 & 173 & 40,9 & \\
\hline Geração não trabalha e nem estuda & & & & & & & 0,034 \\
\hline Não & 1.882 & $74,8(73,0-76,5)$ & 918 & 76,8 & 964 & 73,1 & \\
\hline Sim & 633 & $25,2(23,5-27,0)$ & 278 & 23,3 & 355 & 26,9 & \\
\hline Tem alguma religião ou culto & & & & & & & $<0,001$ \\
\hline Não & 749 & $29,8(28,0-31,6)$ & 403 & 33,7 & 346 & 26,2 & \\
\hline Sim & 1.766 & $70,2(68,4-72,0)$ & 793 & 66,3 & 973 & 73,8 & \\
\hline Cor da pele * & & & & & & & 0,631 \\
\hline Branca & 495 & $19,8(18,3-21,4)$ & 224 & 18,8 & 271 & 20,7 & \\
\hline Preta/Negra & 416 & $16,6(15,2-18,1)$ & 195 & 16,4 & 221 & 16,9 & \\
\hline Parda/Mulata/Cabocla/Morena & 1.589 & $63,6(61,7-65,5)$ & 771 & 64,8 & 818 & 62,4 & \\
\hline Critérios de Classificação Econômica do Brasil * & & & & & & & $<0,001$ \\
\hline A & 94 & $4,2(3,4-5,1)$ & 48 & 4,6 & 46 & 3,9 & \\
\hline B1 & 156 & $6,9(5,9-8,1)$ & 77 & 7,4 & 79 & 6,7 & \\
\hline B2 & 410 & $18,3(16,7-20,0)$ & 210 & 20,1 & 200 & 17,0 & \\
\hline $\mathrm{C} 1$ & 519 & $23,3(21,6-25,1)$ & 258 & 24,6 & 261 & 22,1 & \\
\hline $\mathrm{C} 2$ & 597 & $26,8(25,0-28,7)$ & 287 & 27,4 & 310 & 26,3 & \\
\hline $\mathrm{D} / \mathrm{E}$ & 450 & $20,2(18,6-21,9)$ & 167 & 15,9 & 283 & 24,0 & \\
\hline Pais separados ou divorciados & & & & & & & 0,663 \\
\hline Não & 1.290 & $51,3(49,3-53,3)$ & 608 & 50,8 & 682 & 51,7 & \\
\hline Sim & 1.225 & $48,7(46,7-50,7)$ & 588 & 49,2 & 637 & 48,3 & \\
\hline
\end{tabular}

(continua) 
Tabela 1 (continuação)

\begin{tabular}{|c|c|c|c|c|c|c|c|}
\hline \multirow[t]{2}{*}{ Variáveis } & \multicolumn{2}{|c|}{ Total } & \multicolumn{2}{|c|}{ Masculino } & \multicolumn{2}{|c|}{ Feminino } & \multirow[t]{2}{*}{ Valor de $p$} \\
\hline & $\mathbf{n}$ & $\%(I C 95 \%)$ & $\mathbf{n}$ & $\%$ & $\mathbf{n}$ & $\%$ & \\
\hline Chefe da família & & & & & & & 0,006 \\
\hline Pai & 1.013 & $40,3(38,4-42,3)$ & 504 & 42,1 & 509 & 38,6 & \\
\hline Mãe & 827 & $32,9(31,0-34,8)$ & 360 & 30,1 & 467 & 35,4 & \\
\hline Avós & 294 & $11,7(10,5-13,0)$ & 156 & 13,1 & 138 & 10,6 & \\
\hline Próprio adolescente & 37 & $1,5(1,1-2,1)$ & 22 & 1,8 & 15 & 1,1 & \\
\hline Outro & 344 & $13,6(12,3-15,0)$ & 154 & 12,9 & 189 & 14,3 & \\
\hline Tem plano de saúde & & & & & & & 0,483 \\
\hline Não & 2.006 & $79,8(78,2-81,4)$ & 961 & 80,4 & 1.045 & 79,2 & \\
\hline Sim & 509 & $20,2(18,6-21,8)$ & 235 & 19,6 & 274 & 20,8 & \\
\hline Assaltado alguma vez * & & & & & & & 0,001 \\
\hline Não & 995 & $39,7(37,8-41,7)$ & 432 & 36,3 & 563 & 42,8 & \\
\hline Sim & 1.508 & $60,3(58,3-62,2)$ & 757 & 63,7 & 751 & 57,2 & \\
\hline Alguma vez sentiu insegurança no bairro * & & & & & & & $<0,001$ \\
\hline Não & 650 & $26,0(24,3-27,8)$ & 406 & 34,1 & 244 & 18,6 & \\
\hline Sim & 1.852 & $74,0(72,2-75,7)$ & 783 & 65,9 & 1.070 & 81,4 & \\
\hline Total & 2.515 & 100,0 & 1.196 & 100,0 & 1.319 & 100,0 & \\
\hline
\end{tabular}

IC95\%: intervalo de confiança de 95\%.

* Houve excluídos ou ignorados.

Embora 8,8\% tenham relatado hábito de fumar no passado, apenas 3,6\% fumavam atualmente; e 16,3\% já tinham usado e 5,2\% usavam drogas ilícitas no momento da entrevista. Em relação ao consumo de álcool, 15,7\% apresentaram padrão de consumo de risco, 2,4\% consumo nocivo e 1,3\% era provavelmente dependente. Para todos os padrões de consumo de drogas lícitas e ilícitas o percentual foi maior entre meninos; 23,3\% faziam uso de bebidas energéticas. (Tabela 2).

Dentre os adolescentes, 15,3\% apresentaram QI abaixo da média, sem diferença entre os sexos. Metade dos adolescentes (53,7\%) tinha qualidade do sono ruim, 36,8\% tinham sonolência diurna excessiva e 40,8\% relataram dores de cabeça frequentes. Para todas essas variáveis as prevalências foram maiores em meninas. Relataram tempo de tela $\geq 5$ horas $34,4 \%$, sem diferença entre os sexos (Tabela 2).

Em relação aos transtornos psiquiátricos avaliados, todos foram mais prevalentes nas meninas: depressão (16,8\% vs. 6,4\%), transtorno bipolar (13,8\% vs. 9,8\%), transtorno de ansiedade generalizada atual (4,6\% vs. 2,2\%) e alto risco de suicídio (5,6\% vs. 2,4\%) (Tabela 3).

Na Tabela 4, são apresentadas as informações sobre antropometria, composição corporal e hipertensão dos adolescentes. Foram verificadas diferenças estatisticamente significantes nas prevalências do percentual de gordura corporal elevada, sobrepeso e obesidade, hipertensão arterial e sedentarismo entre os sexos. Pelo IMC, os adolescentes apresentaram 3,7\% de baixo peso, 14,6\% de sobrepeso e $6 \%$ de obesidade. As adolescentes eram as mais obesas $(7,1 \%$ vs. $4,8 \%)$ e tinham mais sobrepeso $(16 \%$ vs. $13 \%$ ) do que os do sexo masculino. Pelo percentual de gordura corporal, estimado com base em pletismografia por deslocamento de ar, 7,8\% apresentaram percentual muito baixo e $24,3 \%$ alto ou muito alto. Os adolescentes do sexo masculino registraram as maiores prevalências de percentual de gordura muito baixo (13,9\% vs. 2,3\%), já as do sexo feminino apresentaram as maiores prevalências de percentual alto ou muito alto (37,3\% vs. 10\%). A prevalência de hipertensão foi de $12 \%$, sendo $21,2 \%$ em homens e 3,4\% em mulheres. Em relação à atividade física, 61,8\% não atenderam à recomendação de praticar pelo menos 75 minutos de atividade física intensa ou 150 de atividade moderada por semana. Os percentuais de adolescentes que não atenderam à recomendação foram maiores no sexo feminino (80,7\% vs. 40,9\%, $\mathrm{p}<0,001)$. 
Tabela 2

Prevalência de comportamentos de risco relativos ao estilo de vida, Quociente de Inteligência e dor de cabeça dos adolescentes da Coorte RPS (consórcio de coortes de nascimento brasileiras em Ribeirão Preto, Pelotas e São Luís) segundo o sexo. São Luís, Maranhão, Brasil, 2016.

\begin{tabular}{|c|c|c|c|c|c|c|c|}
\hline \multirow[t]{2}{*}{ Variáveis } & \multicolumn{2}{|c|}{ Total } & \multicolumn{2}{|c|}{ Masculino } & \multicolumn{2}{|c|}{ Feminino } & \multirow[t]{2}{*}{ Valor de $p$} \\
\hline & $\mathbf{n}$ & $\%$ (IC95\%) & $\mathbf{n}$ & $\%$ & n & $\%$ & \\
\hline Hábito de fumar passado * & & & & & & & $<0,001$ \\
\hline Não & 2.283 & $91,2(90,0-92,3)$ & 1.045 & 87,9 & 1.238 & 94,2 & \\
\hline Sim & 220 & $8,8(7,7-10,0)$ & 144 & 12,1 & 76 & 5,8 & \\
\hline Hábito de fumar atual * & & & & & & & $<0,001$ \\
\hline Não & 2.414 & $96,4(95,6-97,1)$ & 1.127 & 94,8 & 1.287 & 97,9 & \\
\hline Sim & 89 & $3,6(2,9-4,4)$ & 62 & 5,2 & 27 & 2,1 & \\
\hline Uso de drogas ilícitas * & & & & & & & $<0,001$ \\
\hline Nunca usou & 2.037 & $81,9(80,3-83,4)$ & 913 & 77,4 & 1.124 & 86,0 & \\
\hline Já usou & 322 & $12,9(11,6-14,3)$ & 193 & 16,3 & 129 & 9,9 & \\
\hline Usa atualmente & 128 & $5,2(4,3-6,1)$ & 74 & 6,3 & 54 & 4,1 & \\
\hline Consumo de álcool (AUDIT) ** & & & & & & & $<0,001$ \\
\hline Baixo/abstinência & 2.026 & $80,6(79,0-82,1)$ & 905 & 75,7 & 1.121 & 85,0 & \\
\hline Consumo de risco & 395 & $15,7(14,3-17,2)$ & 233 & 19,5 & 162 & & \\
\hline Consumo nocivo & 60 & $2,4(1,8-3,1)$ & 37 & 3,1 & 23 & & \\
\hline Provavelmente dependente & 34 & $1,3(0,9-1,8)$ & 21 & 1,7 & 13 & 15,0 & \\
\hline Uso de bebidas energéticas * & & & & & & & $<0,001$ \\
\hline Não & 1.920 & $76,7(75,0-78,3)$ & 874 & 73,6 & 1.046 & 79,6 & \\
\hline Sim & 582 & $23,3(21,7-25,0)$ & 314 & 26,4 & 268 & 20,4 & \\
\hline Dor de cabeça * & & & & & & & $<0,001$ \\
\hline Não & 1.487 & $59,2(57,3-61,1)$ & 910 & 76,2 & 577 & 43,7 & \\
\hline Sim & 1.026 & $40,8(38,9-42,7)$ & 284 & 23,9 & 742 & 56,3 & \\
\hline Quociente de inteligência total $*, \star \star *$ & & & & & & & 0,058 \\
\hline Acima da média & 575 & $25,4(23,6-27,2)$ & 300 & 27,5 & 275 & 23,5 & \\
\hline Inteligência média & 1.339 & $59,3(57,2-61,3)$ & 620 & 56,9 & 719 & 61,5 & \\
\hline Abaixo da média & 346 & $15,3(13,8-16,9)$ & 170 & 15,6 & 176 & 15,0 & \\
\hline Qualidade do sono (Pittsburgh) *,\# & & & & & & & $<0,001$ \\
\hline Boa & 979 & $46,3(44,2-48,5)$ & 527 & 52,7 & 452 & 40,5 & \\
\hline Ruim & 1.137 & $53,7(41,5-55,8)$ & 472 & 47,3 & 665 & 59,5 & \\
\hline Sonolência diurna excessiva * & & & & & & & $<0,001$ \\
\hline Normal & 1.586 & $63,2(61,3-65,1)$ & 809 & 67,9 & 777 & 58,9 & \\
\hline Presença de sonolência & 924 & $36,8(34,9-38,7)$ & 383 & 32,1 & 541 & 41,1 & \\
\hline Tempo de tela (em horas) * & & & & & & & 0,553 \\
\hline$<2$ horas & 945 & $37,8(35,9-39,7)$ & 448 & 37,7 & 497 & 37,9 & \\
\hline $2-4,9$ & 698 & $27,9(26,1-29,7)$ & 343 & 28,9 & 355 & 27,1 & \\
\hline $5+$ & 856 & $34,3(32,4-36,2)$ & 397 & 33,4 & 459 & 35,0 & \\
\hline Total & 2.515 & 100,0 & 1.196 & 100,0 & 1.319 & 100,0 & \\
\hline
\end{tabular}

IC95\%: intervalo de 95\% de confiança.

* Excluídos os ignorados.

** Utilizado Alcohol Use Disorder Identification Test (AUDIT);

*** Utilizado Weschler Adult Intelligence Scale - WAIS III;

\# Utilizado Índice de Qualidade de Sono de Pittsburgh (IQSP). 
Tabela 3

Prevalência de transtornos psiquiátricos entre adolescentes da Coorte RPS (consórcio de coortes de nascimento brasileiras em Ribeirão Preto, Pelotas e São Luís). São Luís, Maranhão, Brasil, 2016.

\begin{tabular}{|c|c|c|c|c|c|c|c|}
\hline \multirow[t]{2}{*}{ Variáveis } & \multicolumn{2}{|r|}{ Total } & \multicolumn{2}{|c|}{ Masculino } & \multicolumn{2}{|c|}{ Feminino } & \multirow[t]{2}{*}{ Valor de $\mathrm{p}$} \\
\hline & $\mathbf{n}$ & \% (IC95\%) & $\mathbf{n}$ & $\%$ & $\mathbf{n}$ & $\%$ & \\
\hline Risco de suicídio & & & & & & & $<0,001$ \\
\hline Baixo & 2.376 & $94,5(93,5-95,4)$ & 1.156 & 96,7 & 1.220 & 92,5 & \\
\hline Moderado & 36 & $1,4(0,1-1,9)$ & 11 & 0,9 & 25 & 1,9 & \\
\hline Alto & 103 & $4,1(3,4-4,9)$ & 29 & 2,4 & 74 & 5,6 & \\
\hline Depressão * & & & & & & & $<0,001$ \\
\hline Não & 2.204 & $88,2(86,8-89,4)$ & 1.112 & 93,6 & 1.092 & 83,2 & \\
\hline Sim & 296 & $11,8(10,6-13,2)$ & 76 & 6,4 & 220 & 16,8 & \\
\hline Transtorno bipolar & & & & & & & 0,002 \\
\hline Não & 2.203 & $88,1(86,8-89,4)$ & 1.072 & 90,2 & 1.131 & 86,2 & \\
\hline Sim & 297 & $11,8(10,6-13,1)$ & 116 & 9,8 & 181 & 13,8 & \\
\hline Transtorno de ansiedade & & & & & & & 0,001 \\
\hline Não & 2.428 & $96,5(95,7-97,2)$ & 1.170 & 97,8 & 1.258 & 95,4 & \\
\hline Sim & 87 & $3,5(2,8-4,3)$ & 84 & 2,2 & 61 & 4,6 & \\
\hline
\end{tabular}

IC95\%: intervalo de 95\% de confiança.

* Excluídos ou ignorados.

\section{Tabela 4}

Composição corporal, índice de massa corporal (IMC), pressão arterial e atividade física dos adolescentes da Coorte RPS (consórcio de coortes de nascimento brasileiras em Ribeirão Preto, Pelotas e São Luís). São Luís, Maranhão, Brasil, 2016.

\begin{tabular}{|c|c|c|c|c|c|c|c|}
\hline \multirow[t]{2}{*}{ Variáveis } & \multicolumn{2}{|r|}{ Total } & \multicolumn{2}{|c|}{ Masculino } & \multicolumn{2}{|c|}{ Feminino } & \multirow[t]{2}{*}{ Valor de p } \\
\hline & $\mathbf{n}$ & $\%(I C 95 \%)$ & $\mathbf{n}$ & $\%$ & $\mathbf{n}$ & $\%$ & \\
\hline IMC/Idade & & & & & & & 0,008 \\
\hline Adequado & 1.905 & $75,7(74,0-77,4)$ & 935 & 78,2 & 970 & 73,5 & \\
\hline Baixo peso & 93 & $3,7(3,0-4,5)$ & 48 & 4,0 & 45 & 3,4 & \\
\hline Sobrepeso & 366 & $14,6(13,2-16,0)$ & 155 & 13,0 & 211 & 16,0 & \\
\hline Obeso & 151 & $6,0(5,1-7,0)$ & 58 & 4,8 & 93 & 7,1 & \\
\hline Percentual de gordura corporal ** & & & & & & & $<0,001$ \\
\hline Muito baixo & 197 & $7,8(6,8-8,9)$ & 167 & 13,9 & 30 & 2,3 & \\
\hline Baixo & 371 & $14,8(13,4-16,2)$ & 324 & 27,1 & 47 & 3,6 & \\
\hline Ótimo & 1.014 & $40,3(38,4-42,3)$ & 494 & 41,3 & 520 & 39,4 & \\
\hline Moderadamente alto & 322 & $12,8(11,5-14,2)$ & 92 & 7,7 & 230 & 17,4 & \\
\hline Alto & 265 & $10,5(9,3-11,8)$ & 56 & 4,7 & 209 & 15,8 & \\
\hline Muito alto & 346 & $13,8(12,5-15,2)$ & 63 & 5,3 & 285 & 21,5 & \\
\hline Hipertensão arterial * & & & & & & & $<0,001$ \\
\hline Não & 2.187 & $88,0(86,7-89,3)$ & 939 & 78,8 & 1.248 & 96,6 & \\
\hline Sim & 297 & $12,0(10,7-13,3)$ & 253 & 21,2 & 44 & 3,4 & \\
\hline Atividade física $\star \star \star ~$ & & & & & & & $<0,001$ \\
\hline Atendem a recomendação & 956 & $38,2(36,3-40,1)$ & 702 & 59,1 & 254 & 19,3 & \\
\hline Não atendem a recomendação & 1.546 & $61,8(59,9-63,7)$ & 486 & 40,9 & 1.060 & 80,7 & \\
\hline
\end{tabular}

IC95\%: intervalo de 95\% de confiança.

* Excluídos os ignorados.

** Percentual de gordura corporal medido por meio de pletismografia por deslocamento de ar e classificado de acordo com Lohman 19;

*** Utilizado Inquérito de Atividade Física adaptado de Self Administered Physical Activity Checklist (SAPAC). 


\section{Discussão}

Os adolescentes de São Luís participantes deste estudo apresentaram alta prevalência de padrão de consumo de álcool de risco, nocivo e provavelmente dependente, de dor de cabeça e qualidade de sono ruim. Adolescentes do sexo feminino eram as mais inativas e apresentaram o maior percentual de gordura corporal alta e muito alta, e os do sexo masculino tiveram a maior prevalência de hipertensão arterial e de gordura corporal muito baixa.

Apesar de termos observado alto percentual de jovens que estudam e/ou trabalham, é importante destacar que a proporção de jovens que não estudam e nem estão no mercado de trabalho, vivenciando o fenômeno "geração nem nem", foi significativa. Os resultados do presente estudo apresentaram um percentual inferior ao encontrado na Pesquisa Nacional por Amostra de Domicílios (PNAD 2015 22,5\%) 24. Jovens sem escola e sem trabalho são expostos ao risco de viver em condições de vulnerabilidade à violência, segundo dados do Índice de Vulnerabilidade Juvenil à Violência (2017) 25.

Neste trabalho foi elevado o percentual de adolescentes cujos pais eram separados. Nunes-Costa 26 explicam que de acordo com o modelo de Troxel \& Matheus, a separação dos pais é um estressor familiar que pode gerar desorganização familiar, além de redução da segurança econômica. Esses fatores associados à vulnerabilidade biológica, familiar, interpessoal e social, contribuem para a desregulação e insegurança emocional da criança, afetando-a no nível afetivo, comportamental e cognitivo. Portanto, é possível que a separação dos pais, especialmente em idade precoce dos filhos, esteja associada à significativa prevalência de problemas de saúde mental nesta amostra.

Outro aspecto que também pode interferir nos indicadores de saúde mental dos adolescentes que foi muito presente entre jovens estudados é a violência comunitária. Moura et al. 27 , em um trabalho realizado com jovens de 15 a 24 anos, em Brasília, encontraram alta prevalência de jovens que haviam sido furtados nos últimos 12 meses (41\%). Nesse mesmo estudo, $87 \%$ dos jovens referiram não se sentirem seguros na cidade onde moravam e $72 \%$ eram bastante ou extremamente preocupados com sua segurança pessoal. Além disso, a prevalência de sentimentos de desespero, ansiedade e depressão, assim como a pior avaliação da saúde ( $20 \%$ vs. $12 \%)$, foi significativamente maior naqueles que já haviam sofrido algum tipo de violência ( $21 \%$ vs. $14 \%)$.

A prevalência de padrão de consumo de álcool de risco, nocivo ou provavelmente dependente foi elevada. Na Pesquisa Nacional de Saúde (PNS 2013), a prevalência de consumo abusivo de álcool (últimos 30 dias) em adultos jovens no Brasil foi de 13,7\% 28. O uso de substâncias psicoativas como o álcool costuma produzir um efeito multiplicador, em que o consumo de uma substância aumenta a probabilidade de consumo de outras. Nesse sentido, nesta amostra de adolescentes, 18,1\% também já experimentaram ou usam drogas ilícitas, sendo similar ao percentual encontrado na PeNSE (Pesquisa Nacional de Saúde Escolar) entre adolescentes de 16 e 17 anos (16,6\%) 29. Considerando a fase precoce da vida desses indivíduos e as consequências do uso excessivo de álcool e de drogas ilícitas, infere-se a necessidade de pensar em medidas mais eficazes para sua prevenção e controle entre os jovens.

As prevalências de tabagismo em cidades da Região Nordeste estão entre as menores do país. $\mathrm{O}$ tabagismo atual no presente trabalho apresentou prevalência semelhante à reportada no estudo ERICA para adolescentes de 12 a 17 anos em São Luís (4,3\%) e na Região Nordeste (4,7\%) 30. Na PNS (2013) 28, a prevalência de tabagismo atual entre adultos jovens de 18 a 24 anos foi de 10,6\%. Chama atenção o percentual de $8,8 \%$ de adolescentes que em algum momento já tiveram o hábito de fumar. Considerando que apenas 3,6\% relataram fumar atualmente, nota-se que houve uma importante taxa de abandono do tabagismo, o que é positivo e pode ser reflexo da expansão de políticas públicas efetivas de controle do tabagismo.

Cerca de um quarto dos adolescentes desta amostra utilizou bebidas energéticas, que podem influenciar negativamente a qualidade do sono. A composição da maioria das marcas comercializadas no Brasil consiste em uma mistura de carboidratos com taurina, cafeína e vitaminas do complexo B. Sabe-se da interferência dos efeitos da cafeína na duração e qualidade do sono 31,32 e na potencialização de sintomas de ansiedade 33 . Não obstante, o consumo de bebidas energéticas também tem sido associado ao uso e abuso de álcool e outras drogas 34,35 . Portanto, é importante que esse consumo seja acompanhado como fator de risco à saúde entre adolescentes.

A ocorrência de dor de cabeça entre os adolescentes foi elevada. A cefaleia é apontada como um fator que pode interferir negativamente na qualidade de vida de crianças e adolescentes. Em revisão 
sistemática realizada por Silva et al. 36, a cefaleia esteve relacionada com distúrbios emocionais, depressão, ansiedade e dificuldades de interação relacionada à família e à escola. Um dos fatores que tem sido associado a dores de cabeça e que apresentou também elevada prevalência no presente estudo foi o tempo de tela. Observou-se que 34,3\% dos adolescentes apresentaram tempo de tela maior ou igual a cinco horas. O elevado tempo de tela também está associado a outros desfechos desfavoráveis à saúde, tais como alterações na composição corporal, maior risco cardiometabólico, menor prática de atividade física, baixa autoestima e problemas comportamentais ${ }^{37}$. Além do tempo de tela, vale destacar que a prevalência de dor de cabeça foi maior em meninas, as quais também tiveram maior prevalência de qualidade do sono ruim e sonolência excessiva. Portanto, a pior qualidade do sono poderia estar associada à ocorrência de dor de cabeça.

Ainda são poucos os estudos nacionais sobre a qualidade do sono, especialmente entre adolescentes. Entre universitários de instituições públicas no sul de Minas Gerais, com média de idades de 20,7 anos, 60,2\% registraram qualidade do sono ruim pelo índice de Pittsburgh 38 . Distúrbios do sono vêm sendo associados a problemas de desenvolvimento cognitivo, distúrbios psíquicos, problemas metabólicos e de excesso de peso, bem como com maior percepção de estresse 39 . Uma metanálise 40 mostrou que a baixa qualidade do sono está associada ao sobrepeso e à obesidade, independentemente da sua duração, em crianças, adolescentes e adultos jovens. Além disso, a associação entre qualidade do sono e desfechos como redução da sensibilidade à insulina, aumento da pressão arterial e problemas de saúde mental (depressão, ansiedade e estresse) também tem sido encontrada 41,42. Portanto, os resultados do presente trabalho alertam para um indicador que ainda é pouco explorado em ações de prevenção da saúde, mas que deve ser observado com maior atenção, pois pode ter alguma contribuição na epidemia de obesidade que cresce no país, bem como na ocorrência de outras doenças crônicas não transmissíveis.

O risco de suicídio apresentou prevalência baixa quando comparado a outros estudos nacionais. Em uma revisão da literatura realizada por Moreira \& Bastos 43 sobre a prevalência e fatores associados ao risco de suicídio, as prevalências em estudos realizados no Brasil variaram de 7,7\% a 36\%. Nessa revisão, os principais fatores associados ao risco de suicídio foram depressão, uso de álcool e drogas, violência física, problemas de relacionamento com os pais, tristeza e solidão. No presente estudo, o uso de álcool e drogas apresentou prevalência elevada, o que pode ter contribuído para aumentar a prevalência de risco de suicídio encontrada. Meninas apresentaram maior risco de suicídio, consistente com o que vem sendo descrito na literatura. Entretanto, é importante destacar que os meninos normalmente apresentam maior prevalência de suicídio consumado, pois usam métodos mais agressivos 44 .

Quanto aos transtornos mentais, as prevalências encontradas diferem de outros trabalhos nacionais. Em um estudo realizado na Região Sul do Brasil, a prevalência de transtorno de ansiedade foi de 26,4\% entre jovens de 18 a 23 anos, portanto, cerca de oito vezes maior do que no presente estudo 45 . Em Pelotas (Rio Grande do Sul), a prevalência de transtorno bipolar foi de 3,9\% 46, quase três vezes menor do que neste trabalho. Nessa mesma cidade, Munhoz et al. 47 encontraram prevalência de $17 \%$ de depressão entre adolescentes de 10 a 19 anos, valor superior ao observado neste estudo (11,8\%). A presença de sintomas psiquiátricos na adolescência está associada ao uso abusivo de álcool e drogas ilícitas, transtornos alimentares e à ocorrência de comportamentos antissociais entre os adolescentes, além da alta probabilidade de recorrência de doenças psiquiátricas na idade adulta 48 .

A prevalência de obesidade neste estudo (avaliada pelo IMC) foi menor do que a observada no âmbito nacional e, especialmente, nas regiões Sul e Sudeste 4. São poucos os estudos de base populacional que tenham avaliado o percentual de gordura corporal de adolescentes no Brasil por meio de métodos mais acurados, o que dificulta a realização de comparações. No presente trabalho, $24,3 \%$ dos adolescentes tinham percentual de gordura corporal alto e muito alto, porém, apenas $6 \%$ foram diagnosticados com obesidade pelo IMC. Isso indica que uma importante parcela de adolescentes no presente estudo apresenta o fenômeno da obesidade de peso normal. O percentual de gordura corporal encontrado em São Luís foi menor do que o reportado em estudos com adolescentes em Curitiba (Paraná, 40,1\%) 49 e Viçosa (Minas Gerais, 41,3\%) 50, mas concordou com estes estudos ao ser maior entre as meninas a prevalência de gordura corporal elevada. A maior prevalência de inatividade física entre as meninas poderia ajudar a explicar a maior prevalência de percentual de gordura corporal elevada no presente estudo. 
A prevalência de hipertensão no presente trabalho (12\%) foi maior do que a encontrada no estudo ERICA para a Região Nordeste (8,4\%) 4. Em Pelotas, no seguimento aos 23 anos da coorte de 1982, a prevalência de hipertensão entre homens foi de 16,4\% e entre mulheres de 5,3\% 51. Provavelmente, as diferenças de prevalências observadas nesses estudos devem-se às distintas faixas etárias estudadas, pois a hipertensão aumenta com o incremento da idade. Diferenças nas prevalências de sobrepeso e obesidade nesses locais também podem interferir nas distintas prevalências de hipertensão arterial observadas.

O percentual de adolescentes que não atenderam às recomendações mínimas de prática de atividade física foi elevada $(61,9 \%)$ e se assemelha ao de outros estudos com amostras semelhantes. De acordo com a PeNSE, no Maranhão, 71,8\% dos adolescentes não praticavam atividade física em níveis adequados 29 . Dados do mesmo estudo apontaram que 64,7\% dos brasileiros entre 18 e 24 anos não atingiram o nível recomendado de prática de atividade física. Em um estudo de base populacional realizado com adultos jovens de São Luís, em 2010, a prevalência de sedentarismo foi de 69,6\% 52. O não atendimento às recomendações para a prática de atividade física representa um risco adicional à saúde desses jovens, uma vez que manter-se ativo está associado à proteção contra uma série de doenças crônicas não transmissíveis e depressão 23 . Meninas apresentaram o maior percentual de não atendimento às recomendações de prática de atividade física, indicando a necessidade de pensar-se em estratégias específicas para promover e motivar esta atividade entre elas.

Como parte de um estudo longitudinal, embora seja um estudo transversal, esses dados apresentam limitações comuns aos estudos de seguimento, como, por exemplo, a perda de representatividade pelas dificuldades em localizar os adolescentes, tendo sido necessária a incorporação de uma coorte retrospectiva. Comparando-se os seguidos com os não acompanhados na coorte original, maiores percentuais de perdas foram observados entre os mais pobres, indivíduos do sexo feminino, mulheres sem companheiro e de menor escolaridade (Tabela 5), o que pode ter contribuído para subestimar as estimativas globais aqui apresentadas, para as variáveis que apresentaram maiores prevalências nestes estratos mais vulneráveis. Quando foi feita a comparação com a coorte original, na coorte retrospectiva houve menor participação de homens, mulheres sem companheiro e mães com escolaridade intermediária (Tabela 6). Dessa forma, a inclusão da coorte retrospectiva contribuiu, provavelmente, para atenuar o viés de seleção, no caso das variáveis sexo e escolaridade, pois as diferenças ocorreram em direções diferentes. Na coorte original as maiores perdas foram para o sexo feminino e mulheres de menor escolaridade, já na coorte retrospectiva houve maior participação exatamente de indivíduos do sexo feminino e mulheres de menor escolaridade.

Outra limitação é a possibilidade de viés de aferição na verificação do uso de drogas ilícitas. O consumo dessas drogas é normalmente subestimado devido ao possível constrangimento em responder a esta questão. Para minimizar essa possibilidade, o questionário relativo a essa questão foi autoaplicado. Além disso, acredita-se que a prevalência encontrada do uso de drogas também pode ter sido subestimada. A maior parte dos participantes foi localizada nas escolas. Sabe-se que usuários de drogas ilícitas têm menor frequência escolar e maiores índices de abandono, o que pode ter provocado viés de seleção. Para as questões relativas ao álcool e fumo, a possibilidade de viés de aferição é reduzida uma vez que são drogas lícitas e socialmente aceitas. Apresentam-se como pontos fortes do estudo o grande tamanho amostral, o uso de instrumentos validados, a utilização de métodos de alta validade para medida de composição corporal, além de ter sido aplicado um questionário diagnóstico para a avaliação da saúde mental. 
Tabela 5

Comparação de algumas características dos indivíduos seguidos e perdidos da coorte de nascimentos 1997/1998. São Luís, Maranhão, Brasil, 2016.

\begin{tabular}{|c|c|c|c|c|c|c|}
\hline \multirow[t]{2}{*}{ Variáveis } & \multirow{2}{*}{$\begin{array}{l}\text { Nascimento } \\
(1997 / 1998)\end{array}$} & \multicolumn{2}{|c|}{ Não seguido } & \multicolumn{2}{|c|}{ Seguido } & \multirow[t]{2}{*}{ Valor de $p$} \\
\hline & & $\mathbf{n}$ & $\%$ & n (coorte original) & $\%$ (coorte original) & \\
\hline Sexo & & & & & & $<0.001$ \\
\hline Masculino & 1.338 & 957 & 71,5 & 381 & 28,5 & \\
\hline Feminino & 1.105 & 873 & 79,0 & 232 & 21,0 & \\
\hline \multicolumn{7}{|l|}{ Situação conjugal } \\
\hline Casada & 704 & 515 & 73,2 & 189 & 26,8 & 0,044 \\
\hline União consensual & 1.146 & 848 & 74,0 & 298 & 26,0 & \\
\hline Sem companheiro & 592 & 466 & 78,7 & 126 & 21,3 & \\
\hline \multicolumn{7}{|l|}{ Renda familiar (salários mínimos) } \\
\hline Até 1 & 401 & 319 & 79,6 & 82 & 20,4 & 0,019 \\
\hline $1,1-2,9$ & 843 & 637 & 75,6 & 206 & 24,4 & \\
\hline 3 e mais & 1.036 & 747 & 72,1 & 289 & 27,9 & \\
\hline Ignorada & 163 & 127 & 77,9 & 36 & 22,1 & \\
\hline Escolaridade (anos de estudo) & & & & & & 0,020 \\
\hline $12+$ & 119 & 89 & 74,8 & 30 & 25,2 & \\
\hline $5-11$ & 1.898 & 1.399 & 73,7 & 499 & 26,3 & \\
\hline $0-4$ & 420 & 337 & 80,2 & 83 & 19,8 & \\
\hline Idade materna (anos) & & & & & & 0,058 \\
\hline$<20$ & 719 & 556 & 77,3 & 163 & 22,7 & \\
\hline $20-34$ & 1.620 & 1.190 & 73,5 & 430 & 26,5 & \\
\hline 35 e mais & 102 & 82 & 80,4 & 20 & 19,6 & \\
\hline Total & 2.443 & 1.830 & 74,9 & 613 & 25,1 & \\
\hline
\end{tabular}

Tabela 6

Comparação de algumas características dos indivíduos das coortes de nascimentos prospectiva original e retrospectiva. São Luís, Maranhão, Brasil, 2016.

\begin{tabular}{|c|c|c|c|c|c|}
\hline \multirow[t]{2}{*}{ Variáveis } & \multicolumn{2}{|c|}{ Nascimento (1997) } & \multicolumn{2}{|c|}{ Coorte retrospectiva } & \multirow[t]{2}{*}{ Valor de $p$} \\
\hline & $\mathbf{n}$ & $\%$ & $\mathbf{n}$ & $\%$ & \\
\hline \multicolumn{6}{|l|}{ Sexo } \\
\hline Masculino & 1.338 & 54,8 & 769 & 42,7 & $<0,001$ \\
\hline Feminino & 1.105 & 45,2 & 1.033 & 57,3 & \\
\hline \multicolumn{6}{|l|}{ Situação conjugal } \\
\hline Casada & 704 & 28,8 & 512 & 28,4 & 0,006 \\
\hline União consensual & 1.146 & 46,9 & 774 & 43,0 & \\
\hline Sem companheiro & 592 & 24,2 & 325 & 18,0 & \\
\hline Escolaridade (anos) & & & & & $<0,001$ \\
\hline $12+$ & 119 & 4,9 & 119 & 6,6 & \\
\hline $5-11$ & 1.898 & 77,7 & 858 & 47,6 & \\
\hline $0-4$ & 420 & 17,2 & 617 & 34,2 & \\
\hline Total & 2.443 & & 1.802 & & \\
\hline
\end{tabular}




\section{Conclusão}

Os indicadores de saúde de adolescentes de 18 e 19 anos de idade, da coorte de nascimentos 1997/1998 em São Luís, evidenciaram elevadas taxas de consumo excessivo de álcool e exposição a drogas ilícitas, bem como elevadas prevalências de transtornos psiquiátricos e da qualidade ruim do sono. Ressaltese a grande diferença no que diz respeito às taxas de gordura corporal elevada, que foram sete vezes maiores nas meninas, e de hipertensão, que foram sete vezes maiores no sexo masculino. Os percentuais de adolescentes sem estudo ou trabalho e com experiência prévia de violência comunitária chamam atenção para a situação de vulnerabilidade em que vivem estes indivíduos.

Há importantes fatores de risco à saúde que aumentam a vulnerabilidade dos adolescentes, especialmente para as doenças e agravos não transmissíveis e transtornos mentais. Essa situação impõe a necessidade de políticas públicas voltadas a essa faixa-etária. A exposição precoce a esses fatores de risco leva ao acometimento cada vez mais cedo dos nossos adolescentes por doenças e agravos à saúde, aumentando a morbidade nesta faixa etária, o que poderá elevar a morbimortalidade em fases futuras da vida.

\section{Colaboradores}

V. M. F. Simões, R. F. L. Batista, M. T. S. S. B. Alves e A. A. M. Silva participaram da concepção e planejamento, análise, interpretação e redação do trabalho, e aprovou a versão final encaminhada. C; C. C. Ribeiro, E. B. A. F. Thomaz e C. A. Carvalho participaram da análise, interpretação e redação do trabalho, e aprovou a versão final encaminhada.

\section{Informações adicionais}

ORCID: Vanda Maria Ferreira Simões (0000-00018351-1348); Rosângela Fernandes Lucena Batista (0000-0002-1529-0165); Maria Teresa Seabra Soares de Britto e Alves (0000-0002-4806-7752); Cecilia Cláudia Costa Ribeiro (0000-0003-0041-7618); Erika Bárbara Abreu Fonseca Thomaz (0000-00034156-4067); Carolina Abreu de Carvalho (00000001-7900-4642); Antônio Augusto Moura da Silva (0000-0003-4968-5138).

\section{Agradecimentos}

Ao Departamento de Ciência e Tecnologia do Ministério da Saúde (DECIT); ao Conselho Nacional de Desenvolvimento Científico e Tecnológico (CNPq); à Fundação de Amparo à Pesquisa e ao Desenvolvimento Científico e Tecnológico do Maranhão (FAPEMA), pelo financiamento; e ao Hospital Universitário da Universidade Federal do Maranhão, pelo apoio na logística da coleta de dados.

\section{Referências}

1. Jacob CM, Baird J, Barker M, Cooper C, Hanson M. The importance of a life course approach to health: chronic disease risk from preconception through adolescence and adulthood. http://www.who.int/lifecourse/publi cations/life-course-approach-to-health.pdf (acessado em 10/Jun/2018).

2. El Achhab Y, El Ammari A, El Kazdouh H, Najdi A, Berraho M, Tachfouti N, et al. Health risk behaviours amongst school adolescents: protocol for a mixed methods study. BMC Public Health 2016; 16:1209.

3. Sichieri R, Cardoso MA. ERICA: Estudo dos Riscos Cardiovasculares em Adolescentes. Rev Saúde Pública 2016; 50 Suppl 1:2s.

4. Bloch KV, Klein CH, Szklo M, Kuschnir MC, Abreu GA, Barufaldi LA, et al. ERICA: prevalences of hypertension and obesity in Brazilian adolescents. Rev Saúde Pública 2016; 50 Suppl $1: 9 \mathrm{~s}$.

5. Faria-Neto JR, Bento VFR, Baena CP, Olandoski M, Gonçalves LGO, Abreu GA, et al. ERICA: prevalência de dislipidemia em adolescentes brasileiros. Rev Saúde Pública 2016; 50 Suppl 1:10s.

6. Lopes CS, Abreu G de A, Santos DF, Menezes PR, Carvalho KMB, Cunha CF, et al. ERICA: prevalence of common mental disorders in Brazilian adolescents. Rev Saúde Pública 2016; 50 Suppl 1:14s.

7. Oliveira JS, Barufaldi LA, Abreu GA, Leal VS, Brunken GS, Vasconcelos SML, et al. ERICA: uso de telas e consumo de refeições e petiscos por adolescentes brasileiros. Rev Saúde Pública 2016; 50 Suppl 1:7s.

8. Cureau FV, Silva TLN da, Bloch KV, Fujimori E, Belfort DR, Carvalho KMB, et al. ERICA: inatividade física no lazer em adolescentes brasileiros. Rev Saúde Pública 2016; 50 Suppl $1: 4$ s. 
9. Coutinho ESF, França-Santos D, Magliano EDS, Bloch KV, Barufaldi LA, Cunha CF, et al. ERICA: padrões de consumo de bebidas alcoólicas em adolescentes brasileiros. Rev Saúde Pública 2016; 50 Suppl 1:8s.

10. Pinto LW, Assis SG. Violência familiar e comunitária em escolares do Município de São Gonçalo, Rio de Janeiro, Brasil. Rev Bras Epidemiol 2013; 16:288-300.

11. Benetti SPC, Gama C, Vitolo M, Silva MB, D’Ávila A, Zavaschi ML. Violência comunitária, exposição às drogas ilícitas e envolvimento com a lei na adolescência. Psico (Porto Alegre) 2006; 37:279-86,

12. Ximenes LF, Assis SG, Pires TO, Avanci JQ. Violência Comunitária e transtorno de estresse pós-traumático em crianças e adolescentes. Psicol Reflex Crit 2013; 26:443-50.

13. Silva AAM, Coimbra LC, Silva RA, Alves MTSSB, Lamy Filho F, Lamy ZC, et al. Perinatal health and mother-child health care in the municipality of São Luís, Maranhão State, Brazil. Cad Saúde Pública 2001; 17:1412-23.

14. Moretti-Pires RO, Corradi-Webster CM. Adaptação e validação do Alcohol Use Disorder Identification Test (AUDIT) para população ribeirinha do interior da Amazônia, Brasil. Cad Saúde Pública 2011; 27:497-509.

15. Amorim P. Mini International Neuropsychiatric Interview (MINI): validação de entrevista breve para diagnóstico de transtornos mentais. Rev Bras Psiquiatr 2000; 22:106-15.

16. Bertolazi AN. Tradução, adaptação cultural e validação de dois instrumentos de avaliação do sono: escala de sonolência de Epworth e índice de qualidade de sono de Pittsburgh. http:// www.lume.ufrgs.br/handle/10183/14041 (acessado em 11/Jun/2018).

17. Passos MHP, Silva HA, Pitangui ACR, Oliveira VMA, Lima AS, Araújo RC. Confiabilidade e validade da versão brasileira do Índice de Qualidade do Sono de Pittsburgh em adolescentes. J Pediatr (Rio J) 2017; 93:200-6.

18. McKenzie K, Sharples P, Murray AL. Validating the Learning Disability Screening Questionnaire against the Weschler Adult Intelligence Scale, fourth edition. Intellect Dev Disabil 2015; 53:301-7.

19. Lohman TG. Applicability of body composition techniques and constants for children and youths. Exerc Sport Sci Rev 1986; 14:325-57.

20. Onis M, Onyango AW, Borghi E, Siyam A, Nishida C, Siekmann J. Development of a WHO growth reference for school-aged children and adolescents children and adolescents. Bull World Health Organ 2007; 85:660-7.

21. Sallis JF, Strikmiller PK, Harsha D, Feldman HA. Validation of interviewer-and-self-administered physical activity checklists for fifth grade students. Med Sci Sports Exerc 1996; 28:840-51.

22. Ainsworth BE, Haskell WL, Leon AS, Jacobs DR, Montoye H, Sallis JF, et al. Compendium of physical activities: classification of energy costs of human physical activities. Med Sci Sports Exerc 1993; 25:71-80.
23. World Health Organization. Global recommendations on physical activity for health. Geneva: World Health Organization; 2011.

24. Instituto Brasileiro de Geografia e Estatística. Pesquisa Nacional por Amostra de Domicílios: síntese de indicadores 2015. https://biblioteca. ibge.gov.br/visualizacao/livros/liv98887.pdf (acessado em 14/Jun/2018).

25. Secretaria de Governo, Presidência da República. Índice de vulnerabilidade juvenil à violência 2017: desigualdade racial, municípios com mais de 100 mil habitantes. São Paulo: Fórum Brasileiro de Segurança Pública; 2017.

26. Nunes-Costa RA, Lamela DJP V, Figueiredo BFC. Adaptação psicossocial e saúde física em crianças de pais separados. J Pediatr (Rio J.) 2009; 85:385-96.

27. Moura LBA, Oliveira C, Vasconcelos AMN. Violências e juventude em um território da Área Metropolitana de Brasília, Brasil: uma abordagem socioespacial. Ciênc Saúde Colet $2015 ; 20: 3395-405$.

28. Instituto Brasileiro de Geografia e Estatística. Pesquisa Nacional de Saúde: percepção do estado de saúde, estilos de vida e doenças crônicas. ftp://ftp.ibge.gov.br/PNS/2013/pns2013. pdf (acessado em 14/Jun/2018).

29. Instituto Brasileiro de Geografia e Estatística. Pesquisa Nacional de Saúde do Escolar: 2015. https://biblioteca.ibge.gov.br/visualizacao/li vros/liv97870.pdf (acessado em 11/Jun/2018).

30. Figueiredo VC, Szklo AS, Costa LC, Kuschnir MCC, Silva TLN, Bloch KV, et al. ERICA: smoking prevalence in Brazilian adolescents. Rev Saúde Pública 2016; 50 Suppl 1:12s.

31. Mwape RK, Mulenga D. Consumption of energy drinks and their effects on sleep quality among students at the Copperbelt University School of Medicine in Zambia. Sleep Disord 2019; 2019:3434507.

32. Marmorstein NR. Interactions between energy drink consumption and sleep problems: associations with Alcohol Use Among Young Adolescents. J Caffeine Res 2017; 7:111-6.

33. Mangerud WL, Bjerkeset O, Holmen TL, Lydersen S, Indredavik MS. Smoking, alcohol consumption, and drug use among adolescents with psychiatric disorders compared with a population-based sample. J Adolesc 2014; 37:1189-99.

34. Roehrs T, Roth T. Sleep, sleepiness, sleep disorders and alcohol use and abuse. Sleep Med Rev 2001; 5:287-97.

35. Wong MM, Brower KJ, Fitzgerald HE, Zucker RA. Sleep problems in early childhood and early onset of alcohol and other drug use in adolescence. Alcohol Clin Exp Res 2004; 28:57887.

36. Silva BRVS, Silva AO, Diniz PRB, Valença MM, Silva LC, Santos CFBF, et al. Cefaleia e a qualidade de vida em adolescentes. Headache Med 2015; 6:19-23. 
37. Carson V, Hunter S, Kuzik N, Gray CE, Poitras VJ, Chaput J-P, et al. Systematic review of sedentary behaviour and health indicators in school-aged children and youth: an update. Appl Physiol Nutr Metab 2016; 41(6 Suppl 3):S240-65.

38. Mesquita G, Reimão R. Quality of sleep among university students: effects of nighttime computer and television use. Arq Neuropsiquiatr 2010; 68:720-5.

39. Schäfer AA, Domingues MR, Dahly DL, Meller FO, Gonçalves H, Wehrmeister FC, et al. Sleep duration trajectories and body composition in adolescents: Prospective Birth Cohort Study. PLoS One 2016; 11:e0152348.

40. Fatima Y, Doi SAR, Mamun AA. Sleep quality and obesity in young subjects: a meta-analysis. Obes Rev 2016; 17:1154-66.

41. Klingenberg L, Chaput J-P, Holmbäck U, Visby T, Jennum P, Nikolic M, et al. Acute sleep restriction reduces insulin sensitivity in adolescent boys. Sleep 2013; 36:1085-90.

42. João KADR, Jesus SN, Carmo C, Pinto P. The impact of sleep quality on the mental health of a non-clinical population. Sleep Med 2018; 46:69-73.

43. Moreira LCO, Bastos PRHO. Prevalência e fatores associados à ideação suicida na adolescência: revisão de literatura. Revista Quadrimestral da Associação Brasileira de Psicologia Escolar e Educacional 2015; 19:445-53.

44. Braga LL, Dell'Aglio DD. Suicídio na adolescência: fatores de risco, depressão e gênero. Contextos Clínicos 2013; 6:2-14.

45. Costa CO, Branco JC, Vieira IS, Souza LDM, Silva RA. Prevalência de ansiedade e fatores associados em adultos. Jornal Brasileiro de Psiquiatria 2019; 68:92-100.
46. Moreira FP, Jansen K, Cardoso TA, Mondin TC, Magalhães PV, Kapczinski F, et al. Metabolic syndrome and psychiatric disorders: a population-based study. Braz J Psychiatry 2019; 41:38-43.

47. Munhoz TN, Santos IS, Matijasevich A. Depression among brazilian adolescents: a crosssectional population-based study. J Affect Disord 2015; 1:281-6.

48. Welsh JW, Knight JR, Hou SS, Malowney M, Schram P, Sherritt L, et al. Association between substance use diagnoses and psychiatric disorders in an adolescent and young adult clinic-based population. J Adolesc Health 2017; 60:648-52.

49. Bozza R, Campos W, Bacil EDA, Barbosa Filho VC, Hardt JM, Silva PM. Fatores sociodemográficos e comportamentais associados à adiposidade corporal em adolescentes. Rev Paul Pediatr 2014; 32:241-6.

50. Faria FR. Estatos hormonal associado a marcadores cardiometabólicos: estudo comparativo entre as três fases da adolescência. Viçosa: Universidade Federal de Viçosa; 2015.

51. Horta RL, Horta BL, Pinheiro RT, Morales B, Strey MN. Tabaco, álcool e outras drogas entre adolescentes em Pelotas, Rio Grande do Sul, Brasil: uma perspectiva de gênero. Cad Saúde Pública 2007; 23:775-83.

52. Barbosa JB, Santos AM, Barbosa MM, Barbosa MM, Carvalho CA, Fonseca PCA, et al. Metabolic syndrome, insulin resistance and other cardiovascular risk factors in university students. Ciênc Saúde Colet 2016; 21:1123-36. 


\section{Abstract}

The study aimed to estimate the prevalence of health indicators among adolescents in São Luis, Maranhão State, Brazil, in 2016. The analysis included sociodemographic conditions, life habits, body composition, sleep quality, physical activity, cognitive performance, and suicide risk in 2,515 adolescents 18 to 19 years of age. The adolescents belonged to the 1997/1998 birth cohort or were included retrospectively using the SINASC (Brazilian Information System on Live Births) database. The adolescents were mainly from economic class C (50.1\%), $69.5 \%$ were in school, $40.3 \%$ were working, and $25.2 \%$ were neither studying nor working; $60.3 \%$ had been robbed, and $48.7 \%$ had parents who were separated or divorced; $19.4 \%$ showed harmful or excessive alcohol consumption or probable addiction, $19.1 \%$ had used or were using illicit drugs, $53.7 \%$ reported poor sleep quality, $40.8 \%$ reported frequent headaches, $34.3 \%$ reported more than five hours of daily screen time, and 4. $1 \%$ showed high suicide risk. Prevalence of high blood pressure was $12 \%$, and $6 \%$ were obese. Girls were more physically inactive (80.7\%) and showed greater percentage of high (15.8\%) and very high body fat (21.5\%), while boys showed greater prevalence of high blood pressure (21.2\%) and lower prevalence of physical inactivity (40.9\%). High prevalence rates of health risk factors increase the adolescents' vulnerability, exposing these individuals earlier to factors leading to diseases and other health problems.

Adolescent; Social Vulnerability; Nutritional Status; Mental Disorders; Sleep

\section{Resumen}

El objetivo fue estimar la prevalencia de indicadores de salud de adolescentes en São Luís, Maranhão, Brasil, en 2016. Se estudiaron condiciones sociodemográficas, hábitos de vida, composición corporal, calidad del sueño, actividad física, habilidad cognitiva y riesgo de suicidio de 2.515 adolescentes con 18 y 19 años. Los adolescentes pertenecían a la cohorte de nacimiento 1997/1998 o se incluyeron, retrospectivamente, utilizando el banco del SINASC (Sistema de Información sobre Nacidos Vivos). Los adolescentes eran, principalmente, de clase económica $C(50,1 \%)$, un $69,5 \%$ estudiaban, un 40,3\% trabajaban y un 25,2\% no estudiaban ni trabajaban; un 60,3\% ya fueron víctimas de asalto y un $48,7 \%$ tenian a los padres separados. Un 19,4\% presentaron un patrón de consumo nocivo, excesivo o probable dependencia de alcohol, Un 19,1\% consumieron o estaban consumiendo drogas ilícitas, un 53,7\% tenían una calidad de sueño mala, un 40,8\% informaron de dolor de cabeza frecuente, un 34,3\% declararon tiempo de TV al día mayor que cinco horas y un 4,1\% presentaban un alto riesgo de suicidio. La prevalencia de hipertensión arterial fue de un 12\% y de obesidad, 6\%. Las niñas eran más inactivas $(80,7 \%)$ y presentaron un mayor porcentaje de grasa corporal alto $(15,8 \%) y$ muy alto (21,5\%), mientras que los niños tuvieron una mayor prevalencia de hipertensión arterial $(21,2 \%)$ y menor prevalencia de inactividad física $(40,9 \%)$. Las elevadas prevalencias de factores de riesgo para la salud aumentan la vulnerabilidad de los adolescentes, exponiendo a estos individuos precozmente a factores que les llevan a sufrir cada vez más pronto enfermedades y problemas de salud.

Adolescente; Vulnerabilidad Social; Estado Nutricional; Trastornos Mentales; Sueño
Recebido em 28/Ago/2019

Versão final reapresentada em 11/Nov/2019

Aprovado em 06/Dez/2019 